$\underline{\xi}=$ 잘

\title{
Combining different treatment philosophies and techniques to reach the desired treatment goal using butterfly system-customizing orthodontic treatment
}

\author{
Lekhadia DR * \\ Dr. Dhaval Ranjit Lekhadia, In Private Practice of Unique Hospital, off ring road, Surat, Gujarat-395002, India \\ * Corresponding author E-mail:drdhavallekhadia@gmail.com
}

\begin{abstract}
This case report describes the orthodontic treatment of an 18-year-old male patient who presented with Straight profile; tongue thrust habit, proclined upper incisors, generalised spacing in upper and lower arches, Katz's class II premolar relation unilaterally, class II canine relation unilaterally with increased overjet and overbite. A butterfly system was used in the treatment combined with frictionless biomechanics in the initial stage of treatment followed by continuous arch mechanics in the later part of treatment. A tongued crib was used to stop the tongue thrust habit along with one elastic swallow exercise. Micro-implant anchorage was used unilaterally in the upper arch for retraction of the entire segment and correction of the unilateral class II canine and premolar relationship. To avoid a dished in profile, a non-extraction treatment was executed. Final corrections of distally tipped canines were achieved using conventional Begg's uprighting auxiliaries in the vertical slots of butterfly system in the finishing stage. The case was finished using bite settling elastics. Total treatment time was 1 year 2 months. Aesthetic and functional goals were achieved satisfactorily with proper selection of biomechanics.
\end{abstract}

Keywords: Sectional Mechanics, Butterfly system, Micro-implant, Tongue crib, vertical slots

\section{Introduction}

Orthodontic practitioners and beginners in orthodontics often have many questions in mind: which philosophy to use? What bracket prescription is appropriate for a particular case? When to use a straight archwire and segmented mechanics? Is it appropriate to combine the various treatment philosophies and techniques to reach the final treatment outcome?

Making an accurate diagnosis of the changes observed in patients is of utmost importance.It is essential that an effective treatment plan is executed in order to treat the malocclusion within a shorter period of time, with as little injuries to protective and supporting tissues as possible. Thus, recommending the most appropriate treatment for a particular malocclusion, regardless of the type of bracket prescription, technique or slot, is what really matters. For this reason, knowing the principles on which each technique is based on, as well as its limitations, is essential (Godeiro CS et al. 2014).

The pre-adjusted edgewise Straight Wire Appliance was introduced in the 1970s (Thickett E et al. 2007). Since then, there have been many suggested modifications to the bracket prescriptions in terms of torque and tip values, often differing by only a few degrees. Over the years, clinicians have considered how bracket design can help achieve these objectives (Andrews LF 1989). Andrews made extensive measurements on untreated excellent occlusions (McLaughlin RP et al.2001).Molar anchorage loss has been shown to occur during the early stages of alignment with preadjusted appliances (Rajesh $\mathrm{M}$ et al. 2014, Su H et al. 2014).Low friction is most advantageous during initial alignment, however, whereas friction is needed for control in finishing and torque expression. If the friction level of a bracket could be adjusted for different treatment stages, orthodontic tooth movement would be more efficient (Chen $S$ et al. 2015).In a conventional pre-adjusted appliance, with the buccal tube positioned parallel to the line of buccal cusps, the passive archwire will lie below the anterior brackets because of the curve of Spee. When the anterior teeth are engaged with a continuous archwire, a counter clockwise tip forward moment will be created on the molar resulting in anchor loss while the anterior teeth are extruded (McLaughlin RP et al.2001).This bite deepening effect can be avoided by using a sectional archwire in the initial stage of treatment (Orton HS \& McDonald F 1985) and micro-implants can be used to reinforce the anchorage as micro-implants can be more efficient than traditional anchorage methods besides making treatments more predictable (Marassi C \&Marassi 2008).Therefore, combining different treatment techniques and philosophies with a desired bracket system can bring about a superior treatment results.

\section{Case 1:}

The present case report showcases the treatment results achieved by combining the different treatment philosophies and techniques using butterfly system(Bowman SJ \&Carano A 2004).

Diagnosis and Treatment plan:

An 18-year-oldmale patient in the permanent dentition presented with the chief complaint of forwardly placed anterior teeth with spacing in between them. Upon extraoral examination, patient had a straight profile, horizontal growth pattern, reduced nasolabial angle; upper midline shifted toward the right by $1 \mathrm{~mm}$, competent lips and non-consonant smile. (Fig. 1-6). 
He was also diagnosed with tongue thrust habit. Upon intraoral examination, he had proclined anteriors, generalized spacing in upper and lower arch, distally tipped canines in upper and lower arches, increased curve of spee, Class I molar relation bilaterally, Katz Class II premolar relation and class II canine relation on the left side and crossbite i.r.t 15, the overjet was $7 \mathrm{~mm}$ and overbite was $40 \%$ (Fig. 7-11).

The finding were confirmed with study models (Fig. 12-16) and pretreatment radiaographs (Fig. 17, 18).
Cephalometric analysis indicated a Class I skeletal pattern, horizontal growth pattern, reduced lower anterior facial height and Proclined upper and lower incisor. (Table 1)

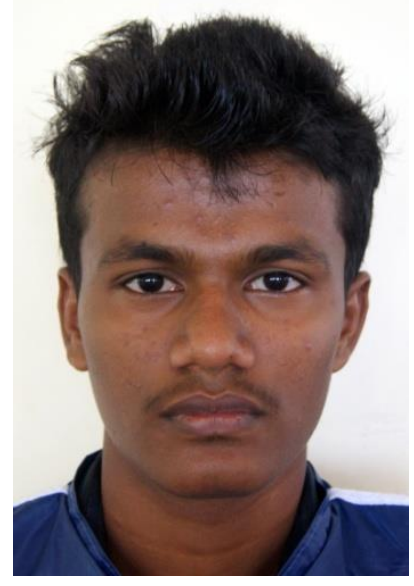

Fig. 1: Pre-Treatment Extraoral Frontal View.

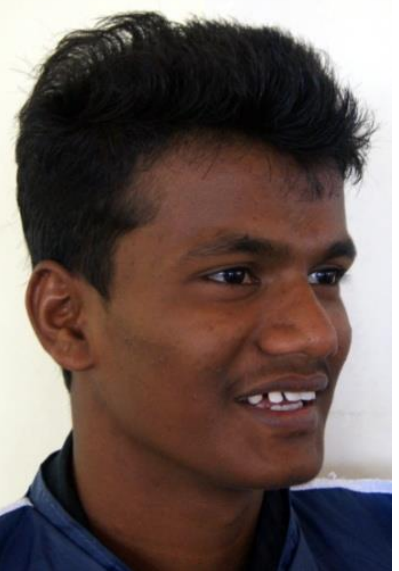

Fig. 4: Pre -Treatment Extraoral Oblique Smile View.

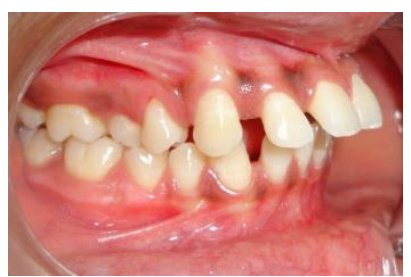

Fig. 7: Pre-Treatment Intraoral Right Lateral View.

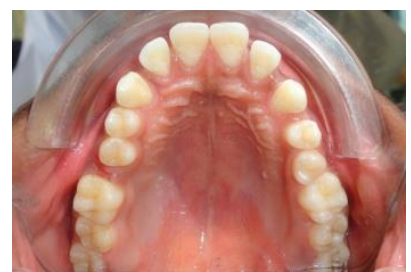

Fig. 10: Pre-Treatment Intraoral Maxillary Occlusal View.

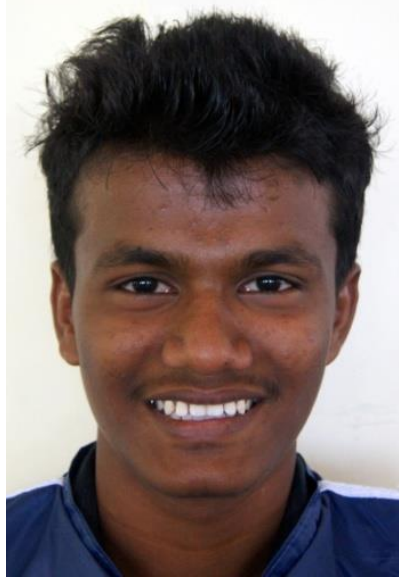

Fig. 2: Pre -Treatment Extraoral Frontal Smile View

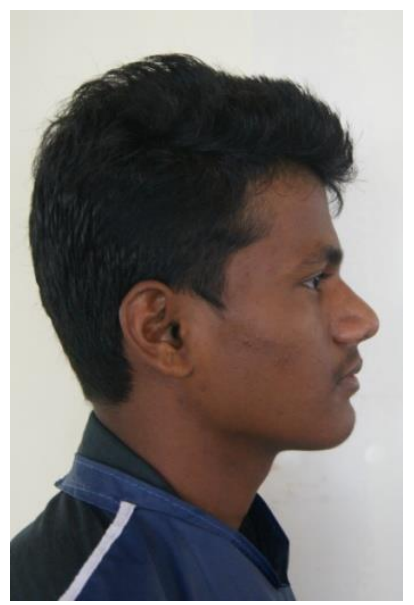

Fig. 5: Pre -Treatment Extraoral Right Lateral Profile View.

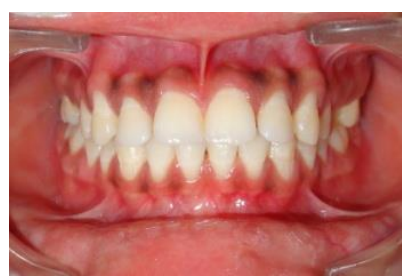

Fig. 8: Pre-Treatment Intraoral Frontal View.

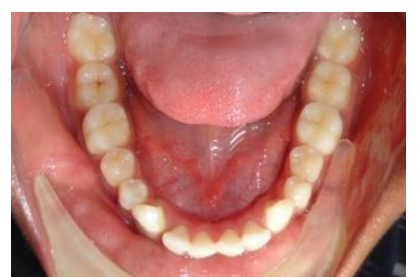

Fig. 11: Pre-Treatment Intraoral Mandibular Occlusal View.

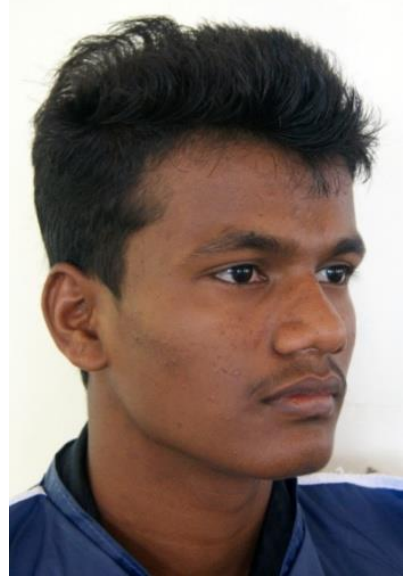

Fig. 3: Pre -Treatment Extraoral Oblique View.

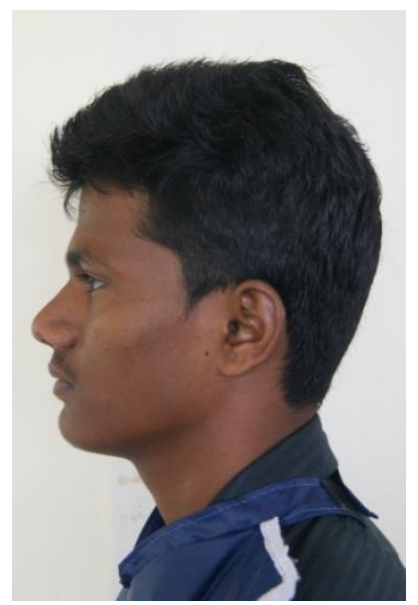

Fig. 6: Pre -Treatment Extraoral Left Lateral Profile View.

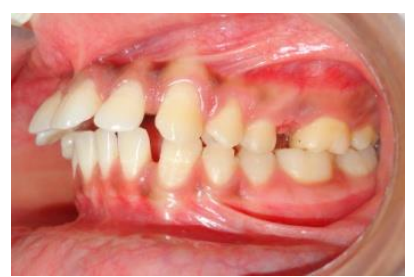

Fig. 9: Pre-Treatment Intraoral Left Lateral View. 


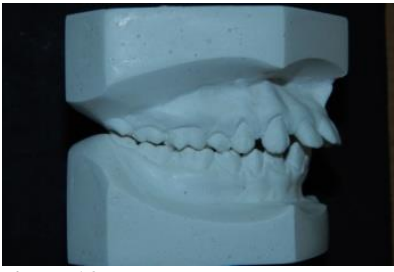

Fig. 12: Pre-Treatment Study Model Right Lateral View.

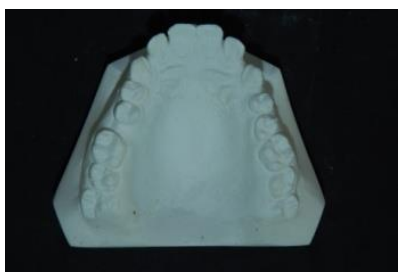

Fig. 15: Pre-Treatment Study Model Maxillary Occlusal View.

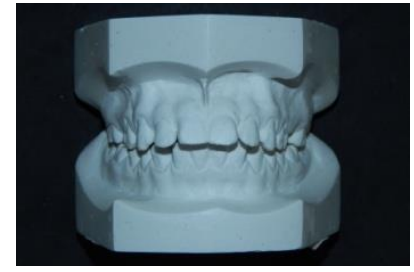

Fig. 13: Pre-Treatment Study Model Frontal View.

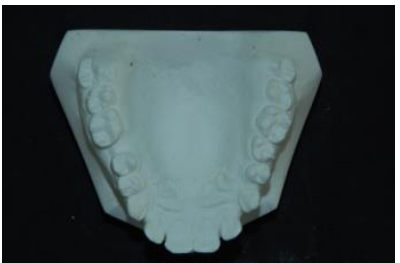

Fig. 16: Pre-Treatment Study Model Mandibular Occlusal View.

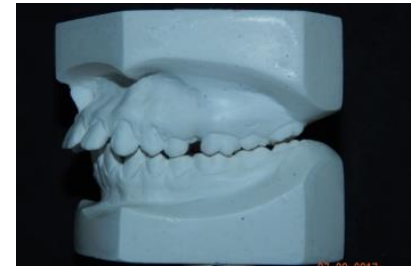

Fig. 14: Pre-Treatment Study Model Left Lateral View.

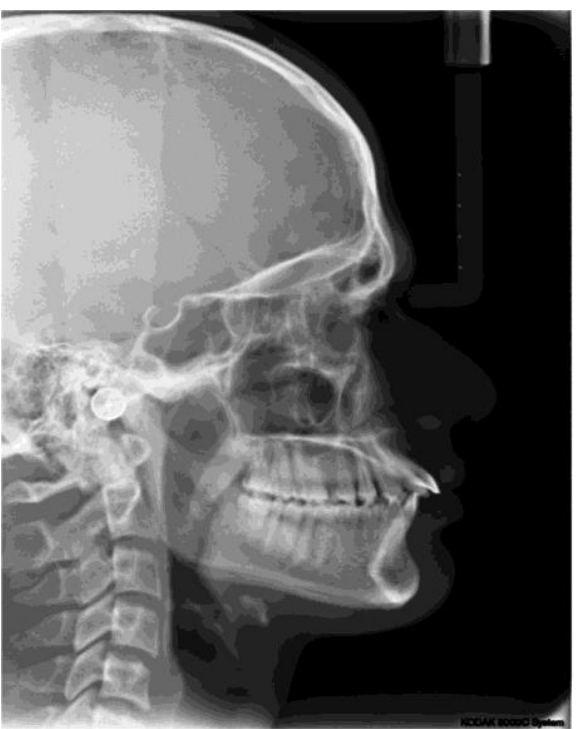

Fig. 17: Pre-Treatment Lateral Cephalogram.

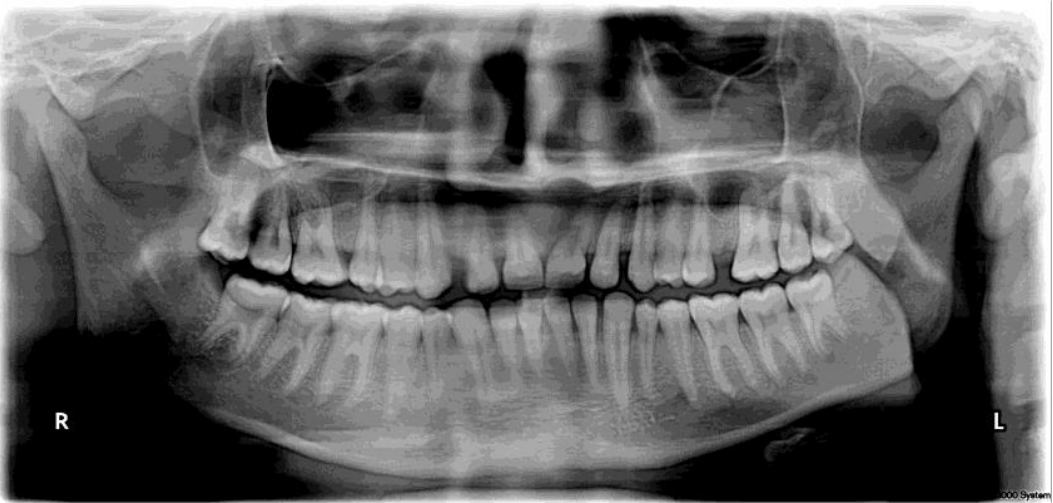

Fig. 18:Pre-Treatment OPG.

Table 1: Cephalometric Values

\begin{tabular}{|c|c|c|}
\hline & Pre-Treatment & Post Treatment \\
\hline SNA & $85^{\circ}$ & $85^{\circ}$ \\
\hline SNB & $83^{\circ}$ & $83^{\circ}$ \\
\hline ANB & $2^{\circ}$ & $2^{\circ}$ \\
\hline Ang.of convexity & $-2^{\circ}$ & $-2^{\circ}$ \\
\hline Wits $\mathrm{AO} / \mathrm{BO}$ & $0 \mathrm{~mm}$ & $0 \mathrm{~mm}$ \\
\hline FMA & $17^{\circ}$ & $19^{\circ}$ \\
\hline SN-GO-GN & $16^{\circ}$ & $18^{\circ}$ \\
\hline Y AXIS & $58^{\circ}$ & $58^{\circ}$ \\
\hline LAFH & $58 \mathrm{~mm}$ & $61 \mathrm{~mm}$ \\
\hline Gonial angle & $121^{\circ}$ & $124^{\circ}$ \\
\hline Base plane ang. & $15^{\circ}$ & $17^{\circ}$ \\
\hline U1 to NA angle & $47^{\circ}$ & $37^{\circ}$ \\
\hline U1 to NA linear & $11 \mathrm{~mm}$ & $6 \mathrm{~mm}$ \\
\hline $\mathrm{U} 1$ to $\mathrm{FH}$ & $135^{\circ}$ & $127^{\circ}$ \\
\hline $\mathrm{U} 1$ to $\mathrm{SN}$ & $130^{\circ}$ & $123^{\circ}$ \\
\hline $\mathrm{L} 1$ to $\mathrm{NB}$ angle & $27^{\circ}$ & $27^{\circ}$ \\
\hline Interincisal angle & $104^{\circ}$ & $114^{\circ}$ \\
\hline Nasolabial angle & $92^{\circ}$ & $110^{\circ}$ \\
\hline 'S' line to upper lip & $0 \mathrm{~mm}$ & $-2 \mathrm{~mm}$ \\
\hline 'S' line to lower lip & $0 \mathrm{~mm}$ & $-2 \mathrm{~mm}$ \\
\hline Lower lip to E-line & $-2 \mathrm{~mm}$ & $-4 \mathrm{~mm}$ \\
\hline
\end{tabular}

Following a comprehensive clinical and data-base analysis, we devised a treatment plan involving non-extraction approach leaving the incisors mildly proclined to prevent a dished in profile and to have an adequate lip support.

\section{Treatment Progress:}

A butterfly system with 0.022 " slot was used. Initial levelling and alignment was carried out in upper and lower arches using sectional 0.016 " Nickel titanium wire from second molar to canine as posterior section and incisors in the anterior section (Fig. 19-23). 


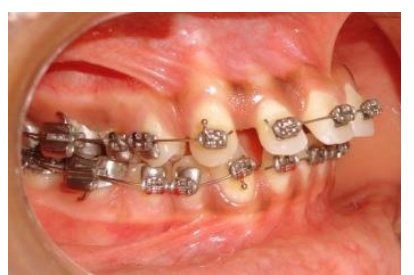

Fig. 19: Initial Levelling and Alignment with Sectional Archwire Right.

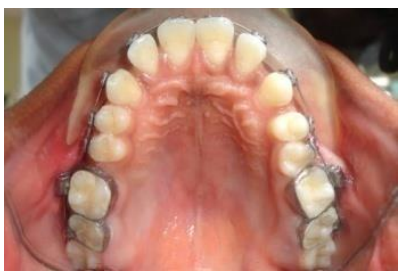

Fig. 22: Initial Levelling and Alignment with Sectional Archwire Maxillary Occlusal View.

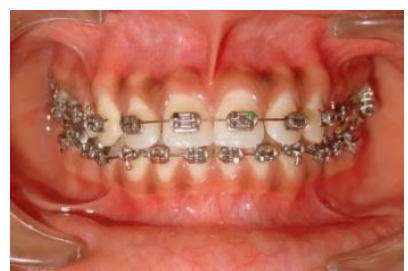

Fig. 20: Initial Levelling and Alignment with Sectional Archwire Frontal View.

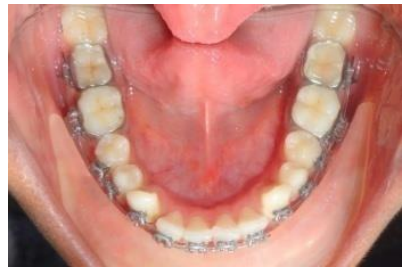

Fig. 23: Initial Levelling and Alignment with Sectional Archwire Mandibular Occlusal View.
This was done to prevent the bite deepening effect of continous arch wire and prevent strain on the anchorage. Myofunctional exercise was advised for the correction of tongue thrust habit. After the correction of distal crown tip of canines, a continuous 0.016 " heat activated nickel titanium wire was place in upper and lower arches. This was followed by $0.019 \times 0.025$ " heat activated nickel titanium wire.

A miniscrew was placed in apical portion, between 2nd premolar and 1st molar, near Cres of posterior segment on class II side (Fig. 24).

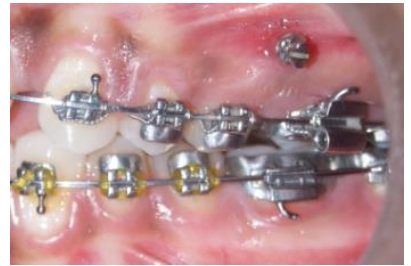

Fig. 24: Micro- Implant on Class II Side in Maxilla.

This assisted us in achieving a Katz class I premolar and a Class I canine relation without disturbing the molars.

Space closure was done on $0.019 \times 0.025$ " stainless steel wires in upper and lower arches using NiTi closed coil springs. Direct anchorage from implants was used on the class II side. A fixed tongue crib was placed because of the persistence of the tongue thrust habit (Fig. 25).

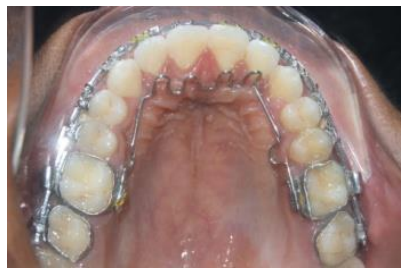

Fig. 25: Fixed Tongue Crib.

An open bite was observed at the end of space closure which was because of the tongue thrust habit. This was corrected using box elastics in the anteriors (Fig. 26) and tongue crib.

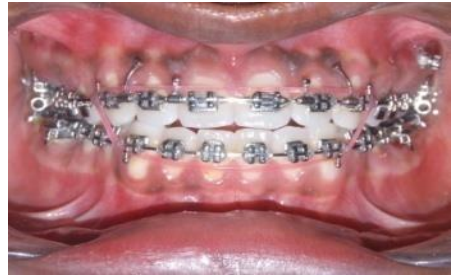

Fig. 26: Box Elastics.
Since the patient did not have enough incisor exposure upon social smile, extrusion of anteriors by box elastics helped achieve a favorable incisor exposure.

At the end of space closure, conventional Begg's uprighting springs were used on upper and lower canines to achieve final correction of the canine tip (Fig. 27-29).

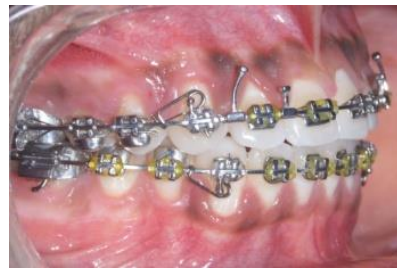

Fig. 27: Traditional Begg's Uprighting Springs Right Lateral View.

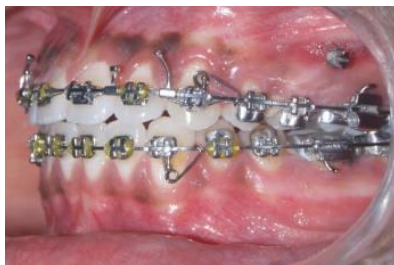

Fig. 28: Traditional Begg's Uprighting Springs Left Lateral View.

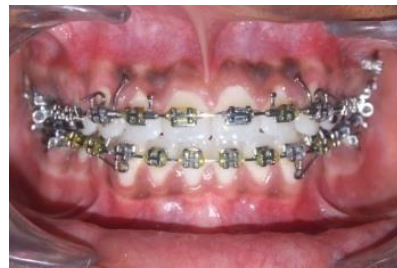

Fig. 29: Traditional Begg's Uprighting Springs Frontal View.

This ensures stability of the treated case. Finishing was done using triangular elastics with hooks in vertical slots on a light 0.016 " NiTi wires in upper and lower arches.

\section{Discussion}

Making an accurate diagnosis of the changes observed in patients and formulating an appropriate treatment plan is the key to successful orthodontic treatment. A preference to butterfly system ${ }^{10}$ was given to treat the present case because it has versatile vertical slots which could be used to upright the canines in the finishing stage of treatment, +3 degrees mesial crown tip which helps to reduce marginal ridge discrepancy especially on the class II side, Progressive mandibular anterior tip which in this case would help 
tent posting of lower incisors and in turn would increase the stability of the end results and preventive mandibular anterior torque of -5 degrees which helps prevent lower anterior flaring and finishing with an adequate overjet without having Bolton's discrepancy. An initial continuous archwire was not used because unlike sectional mechanics, continuous archwires with distally tipped canine crowns tend to deepen the bite and put more strain on the anchorage (McLaughlin RP et al.2001). Training of correct swallow and posture of the tongue was done by myofunctional exercise(Speidel TM et al. 1972) where the patient was guided regarding the correct posture of the tongue during swallowing by various exercises. The patient was asked to place the tip of the tongue in the rugae areas for $5 \mathrm{~min}$ and is asked to swallow and repeat the same with increased frequency. Micro-implant was placed on the Class II side since there was spacing mesial to the molar leaving a weak anchorage value. Maximum anchorage was required on left side since the entire segment had to be retracted in a functional Class I occlusion. The position of micro-implant was preferred in apical

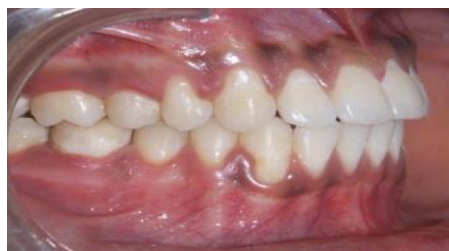

Fig. 30: Post-Treatment Intraoral Right Latera View.

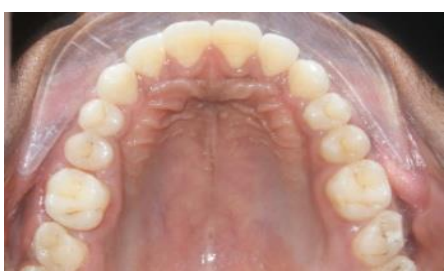

Fig. 33: Post-Treatment Intraoral Maxillary Occlusal View.

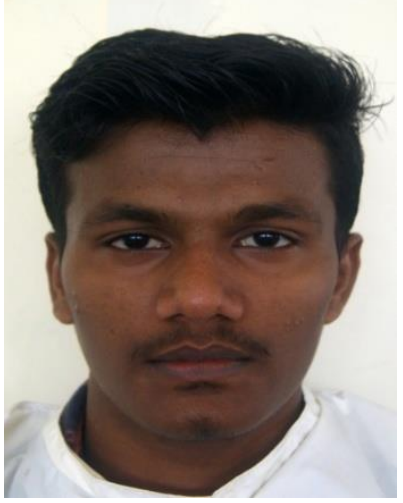

Fig. 35:Post-Treatment Extraoral Frontal View.

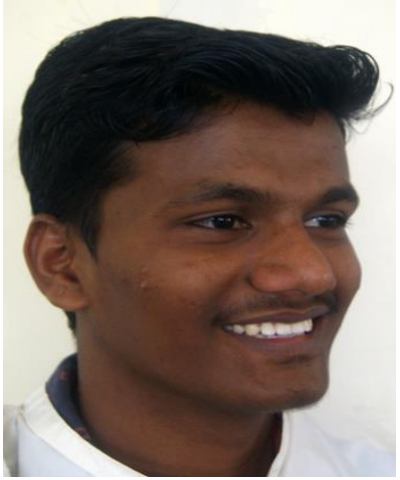

Fig. 38: Post -Treatment Extraoral Oblique Smile View. portion, between 2nd premolar and 1st molar, near Cres of posterior segment. Use of micro-implant for reinforcement of orthodontic anchorage has become increasingly popular in recent years, especially for the space closure in maximum anchorage cases (Park HS et al. 2001). At the end of space closure, traditional Begg's uprighting spring were used in the vertical slots of canine brackets as described by Dr. Bowman SJ (Bowman SJ \&Carano A 2004).

A class I molar relationship was maintained throughout the treatment, Katz class II premolar relation was achieved bilaterally along with bilateral class I canine relation. Overjet was $2 \mathrm{~mm}$ and overbite was $2 \mathrm{~mm}$ and midlines were coincident at the end of the treatment (Fig. 30-34).

An aesthetic, stable and functional occlusion was achieved at the end of the treatment. A consonant smile arc, pleasing soft tissue profile were achieved at the end of treatment (Fig. 35-40).

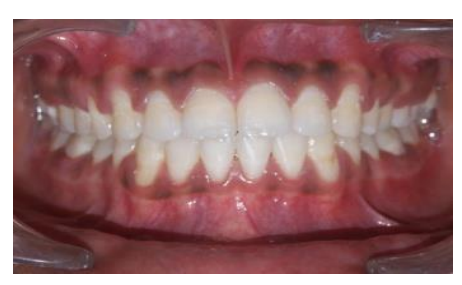

Fig. 31: Post-Treatment Intraoral Frontal View.

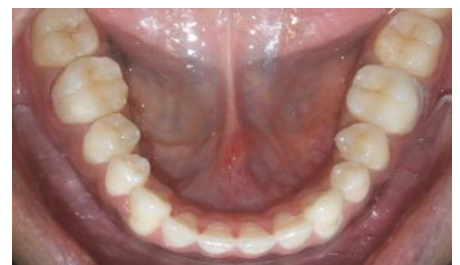

Fig. 34: Post-Treatment Intraoral Mandibular Occlusal View.

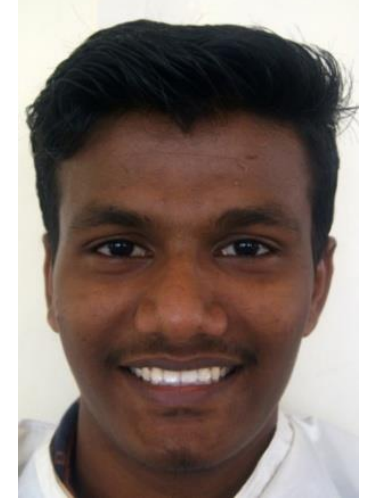

Fig. 36:Post -Treatment Extraoral Frontal Smile View.

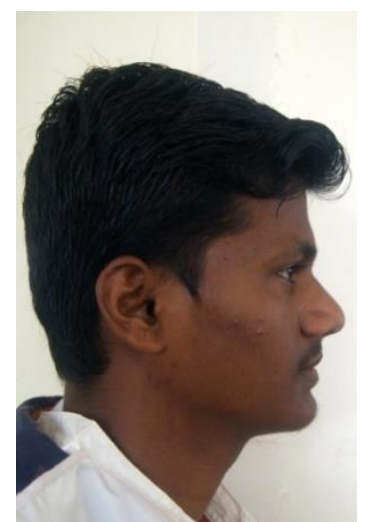

Fig. 39: Post -Treatment Extraoral Right Lateral Profile View.

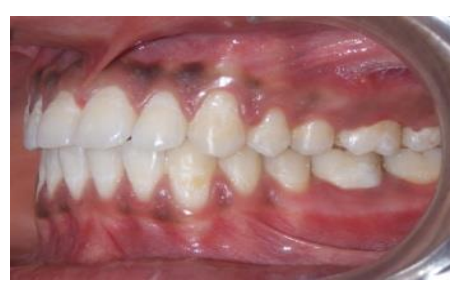

Fig. 32: Post-Treatment Intraoral Left Lateral View.

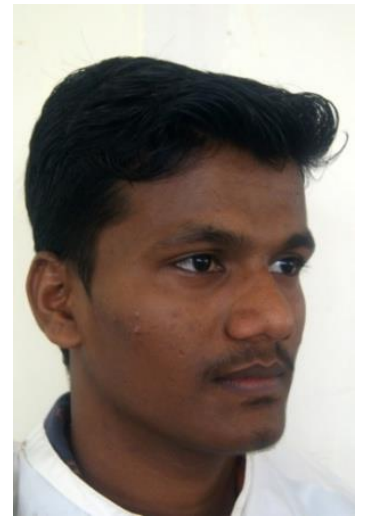

Fig. 37:Post -Treatment Extraoral Oblique View.

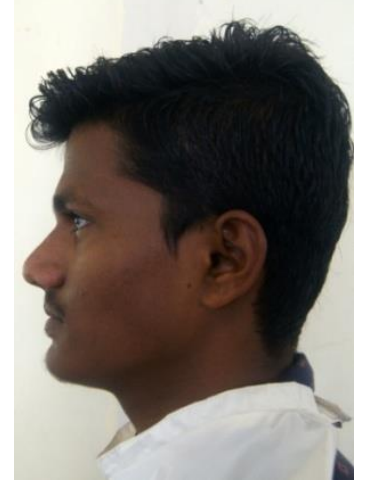

Fig. 40: Post -Treatment Extraoral Left Lateral Profile View. 
Superimposition of pre and post lateral cephalogram reveals that the maxillary incisors were left with enough proclination to provide lip support. The mandibular incisors were finished with adequate tip and torque and mild proclination (Fig. 41).

The post-operative OPG reveals parallel roots without any significant root resorption (Fig.42) and post-operative lateral cephalo- gram reveals adequate lip support and pleasing profile at the end of the treatment (Fig. 43).

The findings were confirmed from post-treatment study models (Fig. 44-48).

Fig. 41: Pre-Post Lateral Cephalogram Superimposition.

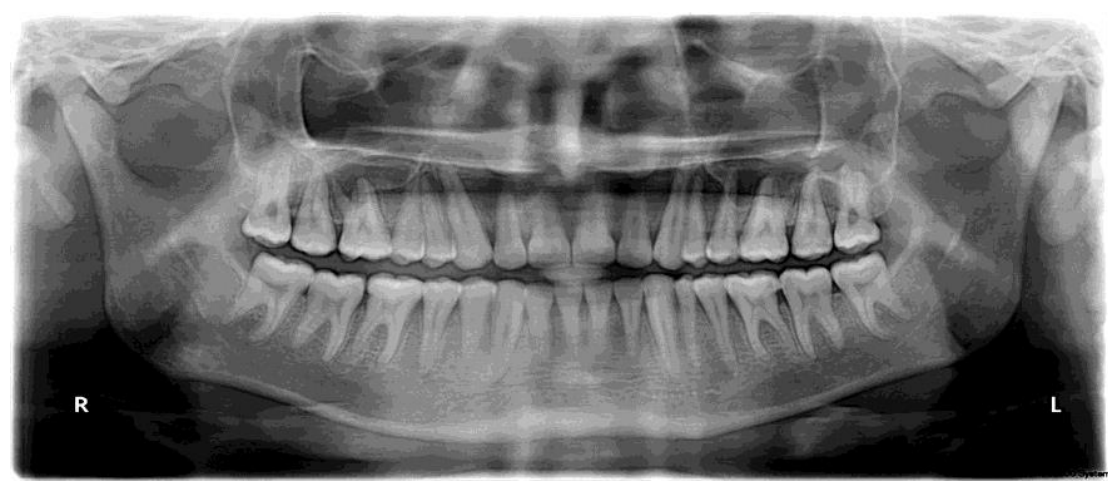

Fig. 42: Post-Treatment OPG.

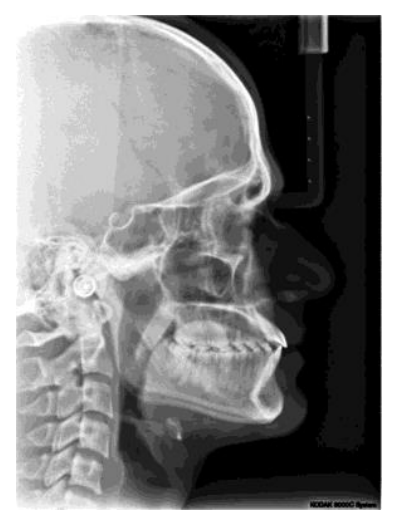

Fig. 43: Post-Treatment Lateral Cephalogram.

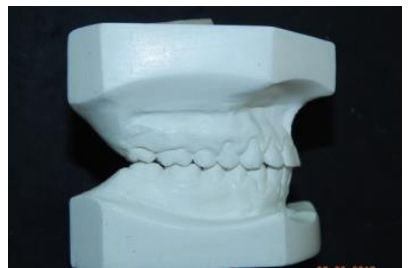

Fig. 44: Post-treatment study model right lateral view.

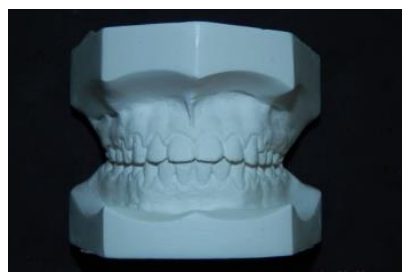

Fig. 45: Post -treatment study model frontal view.

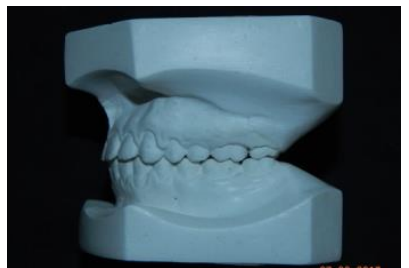

Fig. 46: Post -treatment study model left lateral view. 


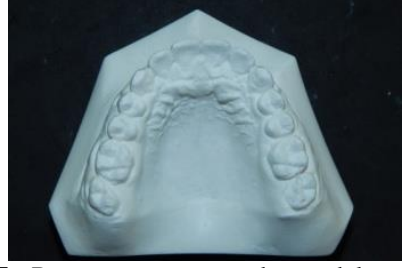

Fig. 47: Post -treatment study model maxillary occlusal view.

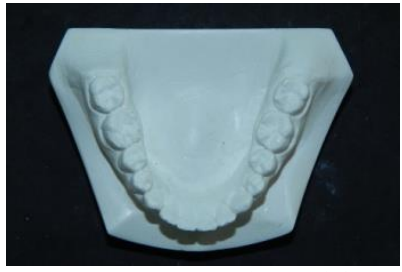

Fig. 48: Post -treatment study model mandibular occlusal view.

\section{Clinical significance}

Use of different techniques in a single case after reaching a proper diagnosis proves to be an efficient treatment mechanic. Unlike the traditional approach of treating all the cases with an MBT or Roth prescription and a continuous archwire mechanics, using a different system and combining and customizing the treatment methods is the need of the hour. Present case report is an evidence of such a method of treating each case differently based on its diagnosis and combining different techniques to suit the demands of a particular malocclusion.

\section{Conclusion}

A careful combination of philosophy, bracket prescription, biomechanics, anchorage reinforcements and finishing auxiliaries can help us to reach the desired treatment outcome. Each malocclusion type requires customizing of treatment method and selection of an appropriate bracket prescription from the pool of bracket systems available. A combination of butterfly system with sectional mechanics in initial stage and continuous wire in the later stage, use of microimplant where necessary and use traditional Beggs'suprighting auxiliaries in the vertical slot of a pre adjusted edgewise appliance have proved to be effective in the present case report.

\section{References}

[1] Godeiro CS, Rabelo F, Antonio RA, Hallissa S, Wilson MA. Segmented arch or continuous arch technique? A rational approach. Dental Press J. Orthod. 2014 Apr; 19 (2): 126141.https://doi.org/10.1590/2176-9451.19.2.126-141.sar.

[2] Thickett E, Taylor NG, Hodge T. Choosing a pre-adjusted orthodontic appliance prescription for anterior teeth. J Orthod 2007; 34:95-100.https://doi.org/10.1179/146531207225021996.

[3] Andrews LF. Straight Wire, the Concept and Appliance. San Diego: L. A. Wells; 1989.

[4] McLaughlin RP, Bennet JC, Trevisi HJ. Systemized Orthodontic Treatment Mechanics. Wolfe, London, Mosby; 2001.https://doi.org/10.1371/journal.pone.0109561.

[5] Rajesh M, Kishore M, Shetty KS. Comparison of anchorage loss following initial leveling and aligning using Roth and MBT prescription-A clinical prospective study. J. Int. Oral Health 2014; 6:16-21.

[6] Su H., Han B, Li S, Na B, Ma W, and Xu, T. Factors predisposing to maxillary anchorage loss: A retrospective study of 1403 cases. PLoS One 9:e10956, 2014.

[7] Chen S, Chen G, Xu T. Clinical Application of the Pass Technique JCO 2015; 43(8):508-515.

[8] Orton HS, McDonald F. A simple sectional canine retraction technique using the properties of nickel titanium rectangular wire. Eur J Orthod 1985;7(2):120-126https://doi.org/10.1093/ejo/7.2.120.

[9] Marassi C, Marassi: Mini- implant assisted anterior retraction. Dental Press J. Orthod.2008; 13 (5): 57-74.

[10] Bowman SJ, Carano A: The Butterfly System. JCO; 38 (5): $274-$ 284.

[11] Speidel TM, Isaacson RJ, Worms FW. Tongue-thrust therapy and anterior dental open-bite. A review of new facial growth data. Am J Orthod 1972; 62:287-95.https://doi.org/10.1016/S00029416(72)90267-9.
[12] H. S. Park, S. M. Bae, H. M. Kyung, and J. H. Sung, "Microimplant anchorage for treatment of skeletal Class I bialveolar protrusion,” Journal of Clinical Orthodontics 2001; 35 (7): 417-422. 